\title{
Intervenções para o TDAH infanto-juvenil que incluem pais como parte do tratamento
}

\author{
Interventions for children and adolescents with ADHD \\ involving parents as part of treatment \\ Intervenciones para el TDAH infanto-juvenil que incluyen \\ padres como parte del tratamiento
}

\author{
Lao Tse Maria Bertoldo* \\ Luan Paris Feijó*** \\ Silvia Pereira da Cruz Benetti***
}

\section{Resumo}

O Transtorno de Déficit de Atenção e Hiperatividade (TDAH) é um diagnóstico frequente em crianças em idade escolar e exige atenção para as consequências no desenvolvimento infantil. Existem diversas linhas de tratamento para o transtorno, especialmente a medicamentosa e a psicossocial. Entretanto, evidências atuais sugerem que a presença de TDAH em crianças pode estar associada a disfuncionalidades na família, relações entre pais e filhos, psicopatologia parental e diminuição da autoeficácia parental. Este artigo de revisão

\footnotetext{
* Graduação em Psicologia pela Universidade Regional do Noroeste do Estado do Rio Grande do Sul, Mestrado em Educação Nas Ciências pela Universidade Regional do Noroeste do Estado do Rio Grande do Sul, formação complementar na área de Desenvolvimento Infantil e Psicopatologia. Professora Horista da Sociedade Educacional Três de Maio, Brasil. Psicóloga clínica. E-mail: laotsebertoldo@yahoo.com.br

** Graduação em Psicologia (Cesuca - Faculdade Inedi), Mestrando em Psicologia - (Unisinos), Especializando em Neuropsicopedagogia (Uniasselvi). Psicólogo clínico. Membro integrante do Núcleo de Estudo e Pesquisa em Psicoterapia Psicanalítica - NEPPP/UNISINOS. Membro associado da Society for Psychotherapy Research (SPR - Capítulo Latino Americano). E-mail: lparisf@gmail.com

*** Graduação em Psicologia pela Universidade Federal do Rio Grande do Sul. Mestrado em Educação pela Universidade Federal do Rio Grande do Sul. Doutorado em Child and Family Studies - Syracuse University. Estágio Sênior na Universidade de Zurich (2014). Professor Adjunto no Programa de Pós-Graduação em Psicologia da Universidade do Vale do Rio dos Sinos, UNISINOS, São Leopoldo, RS. E-mail: spcbenetti@gmail.com
} 
teórica investigou intervenções relacionadas à inclusão de pais como parte do tratamento da criança com TDAH. A literatura analisada relacionou-se a opção e adesão dos pais ao tratamento; estudos que investigaram a eficácia para intervenções com pais como alternativa de tratamento do TDAH nos filhos; e sobre barreiras e acessos às intervenções que os incluem como parte do tratamento da criança com TDAH. Existem significativas evidências de que a inclusão dos pais no tratamento da criança com TDAH traz benefícios para pais e crianças. Pais adquirem habilidades em relação ao conhecimento e manejo do transtorno e aprendem a auxiliar a criança em relação a diferentes aspectos diminuindo sintomas, favorecendo o desenvolvimento infantil e melhorando relações entre pais e filhos.

Palavras-chave: Transtorno do Déficit de Atenção com Hiperatividade; Relações Pais-Filho; Terapêutica

\section{Abstract}

Attention Deficit Hyperactivity Disorder (ADHD) is a frequent diagnosis in school-aged children and requires attention to the consequences on child development. There are several treatment lines for the disorder, especially the drug and psychosocial. However, current evidence suggests that the presence of ADHD in children may be associated with dysfunctionalities in the family, parent-child relationships, parental psychopathology, and decreased parental self-efficacy. This theoretical review article investigated interventions related to parental inclusion as part of the treatment of children with ADHD. The literature analyzed was related to parental choice and adherence to treatment; Studies that have investigated effectiveness for interventions with parents as an alternative treatment of $A D H D$ in children and on barriers and access to parents interventions that include them as part of the treatment of children with $A D H D$. There is significant evidence that the inclusion of parents in the treatment of children with ADHD brings benefits to parents and children. Parents acquire skills in relation to the knowledge and management of the disorder and they learn to help the child in different aspects by reducing symptoms, favoring child development and improving relationships between parents and children.

Keywords: Attention Deficit Disorder with Hyperactivity; Parent-Child Relations; Therapeutics

\section{Resumen}

El trastorno de déficit de atención y la hiperactividad (TDAH) es un diagnóstico frecuente en niños en edad escolar y exige atención a las consecuencias en el desarrollo infantil. Existen diversas líneas de tratamiento para el trastorno, especialmente la medicamentosa y psicosocial. Sin embargo, evidencias actuales sugieren que la presencia de TDAH en niños puede estar asociada a disfuncionalidades en la familia, relaciones entre padres e hijos, psicopatología 
parental y disminución de la autoeficacia parental. Este artículo de revisión teórica investigó intervenciones relacionadas con la inclusión de padres como parte del tratamiento del niño con TDAH. La literatura analizada se relacionó con la opción y adhesión de los padres al tratamiento; Estudios que investigaron la eficacia para intervenciones con padres como alternativa de tratamiento del TDAH en los hijos y sobre barreras y accesos a los padres las intervenciones que los incluyen como parte del tratamiento del niño con TDAH. Existen importantes evidencias de que la inclusión de los padres en el tratamiento del niño con TDAH trae beneficios para padres y niños. Los padres adquieren habilidades en relación al conocimiento y manejo del trastorno y aprenden a auxiliar al niño en relación a diferentes aspectos disminuyendo síntomas, favoreciendo el desarrollo infantil y mejorando relaciones entre padres e hijos.

Palabras clave: Trastorno del Déficit de Atención con Hiperactividad; Relaciones padres-hijo; Terapéutica

O diagnóstico de Transtorno de Déficit de Atenção e Hiperatividade (TDAH) tem sido frequente em crianças em idade escolar e requer atenção para as consequências acarretadas no desenvolvimento infantil. Trata-se de um transtorno diagnosticado mundialmente em larga escala e repetidamente medicado, mesmo sem uma definição consistente no meio científico acerca de sua etiologia (Barkley, 2002; Gusmão, 2009; Leite, 2002; Roskam et al., 2013). O TDAH é considerado um transtorno em função dos diversos prejuízos que acarreta em diferentes aspectos e contextos de vida (Nussbaum, 2015). Tais prejuízos já validados pela literatura através de inúmeros estudos (Dallos \& Smart, 2011; Folquitto, 2013; Johnston \& Mash, 2001; Rohde \& Halpern, 2004) revelam um transtorno com critérios diagnósticos específicos composto de vários sinais e sintomas, havendo necessidade de distingui-lo da existência isolada de sintomas de desatenção e agitação.

Em relação à fenomenologia descritiva, o Manual Diagnóstico e Estatístico dos Transtornos Mentais - DSM 5 (American Psychiatric Association, 2014) estabelece três formas de apresentação para o TDAH definidas de acordo com os sintomas apresentados pela criança: 1) predominantemente desatenta, 2) predominantemente hiperativa-impulsiva, e 3) combinada. Os sintomas de desatenção estão mais relacionados a situações como perder objetos, cometer erros ou descuidos escolares, não conseguir persistir em 
tarefas que envolvam um grande esforço mental continuado ou termina-las. Já os sintomas de hiperatividade manifestam-se na tendência a estar sempre em movimento, na agitação ao falar, na demonstração de impulsividade, entre outros (Rohde \& Halpern, 2004). Para configurar a apresentação combinada de TDAH, o indivíduo deve preencher 6 critérios para a apresentação desatenta e 6 para hiperativa-impulsiva (American Psychiatric Association, 2014).

Além dos sintomas de desatenção e hiperatividade-impulsividade, característicos do transtorno em questão, a maioria das crianças com TDAH apresenta também como sintomas, baixa autoestima e dificuldades de socialização (Barkley, 2002). Assim, os infantes podem vivenciar rejeição no nível de interações sociais, devido ao fato de não se adequarem aos ideais esperados entre seus pares de mesma faixa etária.

Frequentemente algumas características do comportamento da criança com TDAH, como a desatenção ou a agitação, provocam uma reação negativa por parte dos pares nos aspectos afetivo e social. Em situações de coletividade, as crianças podem reagir com mais desatenção ou impulsividade, enquanto que individualmente, com um dos cuidadores, pai, mãe, avós ou professores, apresentam geralmente mais facilidade em se concentrar e responder às demandas, sobretudo quando há adequada condução da situação. Cabe salientar, que os pais, não raro, se irritam com o comportamento das crianças com TDAH em virtude dos sintomas apresentados, no entanto, estes têm um papel importante nas vicissitudes do quadro a partir da posição que adotarem frente ao tratamento (Barkley, 2002).

As possibilidades de tratamento para a criança com TDAH estão centralizadas em duas linhas, as de atendimento psicossocial, compostas por diferentes intervenções terapêuticas ou psicopedagógicas e que visam o atendimento apenas à criança, combinação de atendimento com os pais, ou versões combinadas com medicamentos; e as de caráter exclusivamente medicamentoso, que não incluem outras linhas terapêuticas de atendimento às crianças ou aos pais. Neste estudo, iremos abordar tratamentos psicossociais, sendo estes os que geralmente enfocam os pais como parte das medidas terapêuticas. $\mathrm{O}$ auxílio aos pais, seja pelo viés de treinamento, da psicoeducação ou do manejo comportamental, é fundamental para que 
estes tenham condições de compreender aspectos essenciais do transtorno manifestado por seus filhos e de apoiar cotidianamente a criança na tarefa de organizar o que não é adaptativo.

Mediante os inúmeros prejuízos relacionáveis ao TDAH é necessário refletir sobre e construir alternativas eficazes de intervenções que não sejam reduzidas apenas à administração de medicamentos enquanto via privilegiada de tratamento (Folquitto, 2013). Pesquisas atuais revelam que o tratamento medicamentoso é útil no alívio dos principais sintomas, mas pouco agrega na possibilidade de auxiliar a criança nos demais aspectos do desenvolvimento que sofrem repercussões em decorrência do transtorno (Bronw, 2007).

A supervalorização da alternativa química teria suas raízes em concepções iniciais acerca da etiologia do transtorno, fortemente vinculadas às questões biológicas. Inicialmente havia o entendimento da etiologia como possível lesão cerebral, tese que em seguida foi substituída pelo entendimento de disfunção cerebral, sendo a primeira relacionada à ideia de existência de um marcador biológico, que acabou não se confirmando. Essa concepção sobre o TDAH foi modificada, passando a incluir a noção de uma disfuncionalidade em algumas áreas cerebrais que podem ser acionadas pela dinâmica do quadro clínico (Banaschewski et al., 2017; Caliman, 2010).

Atualmente é consenso que a causa do TDAH é multifatorial, tendo seu desencadeamento e desenvolvimento determinados, inclusive, por situações ambientais, que se aliam a suscetibilidades neurofisiológicas e genéticas (American Psychiatric Association, 2014; García et al., 2008; Moreno Fontiveros, Martínez Vera, Tejada González, González Igeño, \& García Resa, 2015; Murray-Close et al., 2010; Rohde \& Halpern, 2004). Análises empíricas apontam a ansiedade, depressão, conflitos familiares e problemas na parentalidade como possíveis fatores comuns na história clínica de crianças com TDAH e suas famílias. Partindo de tais apontamentos, os estudos sugerem a necessidade de considerar os pais no tratamento da criança (Barkley, 2002; França, 2012; García et al., 2008).

Para Murray-Close et al. (2010), são necessárias intervenções dinâmicas no tratamento do TDAH tendo em vista a gama de sintomas emocionais e de comportamento apresentados pelas crianças afetadas pelo 
transtorno. Segundo estudos, vivências emocionais negativas na primeira infância podem acarretar em prejuízos no desenvolvimento, piorando sintomas iniciais do TDAH, dessa maneira, intervenções voltadas para a restauração da função emocional e social são necessárias como parte do tratamento.

De acordo com pesquisas voltadas para o tema, o desenvolvimento de tratamentos não farmacológicos para o TDAH está sendo considerado uma prioridade por alguns pesquisadores (Pfiffner et al., 2014). Rohde e Halpern (2004) salientam que o tratamento da criança com TDAH deve ter uma abordagem múltipla, envolvendo intervenções com pais e professores como princípio básico de tratamento, tanto em uma perspectiva que envolva intervenções psicológicas e sociais, quanto farmacológicas, quando tal meio for apropriado.

Merece atenção igualmente as condições de saúde mental apresentadas pelos cuidadores da criança. A influência dos pais na origem ou manutenção de psicopatologias nos filhos tem sido amplamente investigada através de estudos que associam oscilações de humor nos pais ao surgimento de quadro depressivo nas crianças, bem como a presença de psicopatologia na mãe e o consumo de drogas em filhos adolescentes. Há, ainda, pesquisas que relacionam crianças com transtorno obsessivo compulsivo (TOC) e pais com personalidade evitativa, obsessivo-compulsiva e transtornos depressivos. Em casos ainda mais extremos, como o do abuso físico da criança por parte dos pais, identificou-se em estudos a repercussão da violência em uma série de problemas emocionais na criança. É neste sentido, compreendendo a importância da configuração da relação familiar, que se acredita que implicar os pais no tratamento do TDAH infantil pode ser efetivo.

Evidências na literatura sugerem que muitas vezes pais de crianças com TDAH podem ter um funcionamento subjetivo diferente de pais de crianças com desenvolvimento típico, podendo apresentar pouca sensibilidade e empatia ou falta de atitude positiva por parte da mãe. Tais características sugerem, segundo pesquisas, associação com maiores níveis de desatenção (Keown, 2012). Já a impulsividade paterna poderia estar relacionada à maior agitação na escola. Ainda que esses estudos não tenham o poder preditivo para apurar se estas características são anteriores ou 
posteriores ao surgimento do TDAH nos filhos, revelam a necessidade de observar as associações entre subjetividade parental e sua influência nos processos de saúde/adoecimento dos filhos.

A partir de tais reflexões sobre o transtorno e seu possível tratamento, o objetivo deste artigo é avaliar estudos que versam sobre a inclusão de pais em intervenções como parte do tratamento de crianças ou adolescentes com TDAH. Assim, procurou-se identificar quais são as modalidades de intervenção usualmente desenvolvidas com os pais, bem como os benefícios e as barreiras apontadas nos estudos analisados.

\section{MÉTODO}

Com o intuito de compreender como diferentes estudos retratam a inclusão dos pais em intervenções como parte do tratamento de crianças ou adolescentes com TDAH, buscou-se realizar uma revisão da literatura a partir de duas bases de dados: o Portal da CAPES e o BVS-Psi. A seleção de artigos foi realizada nos portais CAPES e BVS-Psi fazendo uso dos descritores “ADHD"; "Parents" e "Interventions", em publicações dos últimos dez anos, aplicando-se os filtros de idioma Português, Inglês e Espanhol. No Portal BVS-Psi foi aplicado o filtro de assunto principal "Pais".

No Portal BVS-Psi, foram encontrados 317 documentos, após a aplicação do primeiro filtro, idioma, os artigos que não estavam em Português, Espanhol ou Inglês foram excluídos. Ao todo foram excluídos 9 textos em alemão, 2 textos em chinês, 1 texto em hebraico e 1 texto em Holandês. Restaram 268 artigos e, após a aplicação do filtro, assuntos principais, selecionando o assunto "Pais", restaram 52 artigos, dos quais foram escolhidos para estudo aqueles com publicação datada dos últimos dez anos. Destes, 41 foram analisados por dois juízes em relação à pertinência do tema. Ao final desta análise foram escolhidos 18 artigos do portal BVS-Psi para compor este trabalho. A exclusão de artigos pelos juízes deu-se em virtude de fuga ao tema, o texto versar exclusivamente sobre uso da medicação, apresentar teorizações sobre etiologia, comorbidades, relações profissional-família e outros temas não diretamente relacionados às intervenções com pais no tratamento da criança com TDAH. 
Quanto aos resultados do Portal da CAPES, os descritores foram utilizados na sequência "ADHD" "parents" "interventions", buscando por textos datados dos últimos dez anos, o que resultou em 24 artigos. Destes, 1 encontrava-se indisponível, 2 eram artigos jornalísticos e 4 artigos da busca estavam duplicados. Quinze artigos não apresentavam relação direta com o tema, apresentado considerações sobre doenças físicas ou neurológicas e intervenções paternas sem relação com o TDAH, ou, ainda, tratavam de investigações sobre características gerais do transtorno e seu tratamento farmacológico. Deste portal, 2 artigos foram selecionados para este trabalho e foram incluídos ainda mais quatro artigos das referências dos estudos selecionados.

\section{RESULTADOS}

Os resultados sugerem que existem diferentes linhas de pesquisa sobre a efetividade de tratamentos para o TDAH, incluindo aquelas que consideram intervenções com os pais como parte do tratamento da criança. É frequente que os pais sejam defrontados com a necessidade de optar por alguma linha de tratamento considerada eficaz para o filho e isso muitas vezes ocorre sem que os responsáveis possam contar com as informações necessárias para uma decisão suportada por evidências científicas (Schatz et al., 2015).

Para o tratamento do TDAH, as opções disponíveis figuram desde tratamentos puramente farmacológicos, sem intervenções das quais os pais participem diretamente como parte do tratamento, até aquelas de caráter psicossocial, que incluem os responsáveis pela criança ou adolescente. As categorias de terapêuticas psicossociais relacionam-se ao treinamento de pais, à participação dos mesmos em grupos, fazendo parte de sessões destinadas aos pais individualmente, ou em atividades de caráter psicopedagógico, distinguindo-se das intervenções medicamentosas, que não enfocam aspecto algum da subjetividade ou comportamento (Coates, Taylor \& Sayal, 2015; Daley \& O’Brien, 2013; Mulqueen, Bartley, \& Bloch, 2015; Stattin, Enebrink, Özdemir, \& Giannotta, 2015). 
As propostas de tratamento que incluem os pais são variadas, abarcando desde aquelas em que os pais passam a receber treinamento para habilidades específicas de manejo do TDAH num formato de intervenção dirigido apenas a eles, até aquelas que podem apresentar-se como propostas de uma versão combinada de tratamento para os pais e para as crianças simultaneamente. Em qualquer um destes formatos, intervenções como os programas de treinamento de pais são ações psicossociais destinadas a formar os pais em técnicas que lhes permitam gerenciar o comportamento desadaptativo típico do TDAH apresentado por seus filhos (Webster-Stratton, Reid, \& Beauchaine, 2013; Zwi, Jones, Thorgaard, York, \& Dennis, 2011).

Na maioria das opções de tratamento existentes para o TDAH os pais acabam tendo um papel decisivo quanto à condução da criança, pois, em função dos filhos não possuírem autonomia, são os pais que necessitam decidir qual é a melhor alternativa terapêutica. Esta decisão pode ser influenciada tanto por informações que os responsáveis já possuem sobre o transtorno, como pela ausência destas informações ou, pela orientação recebida de outros profissionais com quem possuem contato e, ainda, de outros pais que tiveram maior ou menor satisfação com um ou outro tipo de tratamento (McGuinness, 2008; Schatz et al., 2015). Essa responsabilidade na decisão quanto ao tratamento da criança deve ser compartilhada (caso a criança conte com o par parental como seus responsáveis), devendo ser estimulada pelos profissionais que realizam os atendimentos à família, já nas primeiras consultas, o que pode repercutir favoravelmente no tratamento da criança (Brinkman et al., 2013).

\section{Opção e adesão dos pais ao tratamento}

Apesar da adesão predominante ao tratamento farmacológico (McGuinness, 2008), algumas medidas permitem aos pais avaliar outras terapêuticas. Isso se dá através da informação que os pais possam dispor inicialmente, das preferências particulares da família em relação aos 
profissionais ou métodos terapêuticos, ou até mesmo pelas condições de saúde mental dos cuidadores ao perceberem e dedicarem-se a alguma opção de tratamento para os filhos (McGuinness, 2008).

Resultados potenciais de diversas intervenções, quando acessadas pelos pais, desempenham um importante papel nas decisões das famílias quanto ao início do tratamento do TDAH. Segundo McGuinness (2008), nos Estados Unidos a medicação é muitas vezes a primeira intervenção utilizada no tratamento de TDAH. Entretanto, intervenções psicossociais com pais e professores que trabalham em conjunto para moldar o comportamento da criança são consideradas alternativas eficazes, permitindo menor quantidade de medicação e, consequentemente, menos efeitos colaterais advindos desta medida.

Todavia, pesquisas ainda são necessárias para entender as particularidades oferecidas pelos tratamentos psicossociais, as preferências por intervenções exclusivas ou combinadas a medicação, bem como para entender o papel dos pais, cuidadores, professores ou outros profissionais que tem contato com a criança neste processo de adesão ao tratamento (McGuinness, 2008). O grau de satisfação relatada por outros pais parece ser claramente um fator que influi na adesão a um ou outro tipo de tratamento pelos pais da criança em início de tratamento. Segundo Schatz et al. (2015) através de revisão sistemática, a procura pelo tratamento medicamentoso é maior, mas muitos pais de crianças com TDAH preferem evitar medicamentos estimulantes em favor de intervenções comportamentais ou psicossociais. Neste sentido, parece não haver ainda muita clareza para os pais sobre o alcance de um tratamento em detrimento ao outro.

Frente a múltiplas opções de tratamento disponíveis, há que se considerar que são diversos os níveis de desenvolvimento e alcance das intervenções, assim como é variado o número de indivíduos potencialmente envolvidos (crianças, pais e adultos com TDAH). Ao avaliar 41 estudos sobre quais são os aspectos do tratamento de indivíduos com TDAH mais estudados, valorizados e determinantes nas decisões de tratamento, Schatz et al. (2015) consideraram que poucos estudos focaram nas preferências de crianças e adolescentes com TDAH em comparação àqueles que se concentram nas preferências dos pais. Além disso, a maioria dos estudos 
se concentrou principalmente em tratamentos medicamentosos, e poucos em tratamentos psicossociais. A pouca valorização dos estudos em relação à investigação das preferências das crianças e adolescentes, assim como a supervalorização do tratamento medicamentoso, indica pouca exploração dos tratamentos psicossociais que incluem pais, bem como evidencia o poder do saber do adulto em detrimento ao próprio saber da criança/ adolescente em relação às preferências no tratamento (Schatz et al., 2015).

A escolha, a adesão ou até mesmo a compreensão de aspectos relacionados ao tratamento da criança pode sofrer interferências importantes quando há existência de psicopatologia nos pais. Pesquisas reconheceram que alguns pais de crianças e adolescentes com TDAH apresentam este mesmo quadro ou outras psicopatologias que podem contribuir para a paternidade inadaptativa e podem também estar relacionadas a dificuldades na administração de medidas destinadas a auxiliar a criança em seu tratamento (Modesto-Lowe, Chaplin, Godsay, \& Soovajian, 2014). Assim, o modo como os pais são auxiliados no início do tratamento também parece repercutir na sua forma de implicação e na adesão às opções disponíveis para o quadro clínico apresentado pelos filhos.

Um estudo realizado por Brinkman et al. (2013) buscou analisar o efeito de uma intervenção de decisão compartilhada com pais de crianças recém-diagnosticadas com TDAH. Sete pediatras participaram de um pré e pós-teste, no qual foi oferecida ajuda para os 33 pais compreenderem o TDAH e desenvolverem um plano de tratamento, intervenção comparada a um grupo controle de 21 pais. A intervenção aumentou a tomada de decisão compartilhada dos pais. A decisão compartilhada certamente implica em melhores resultados no manejo da criança, de modo que ambos os pais sentem-se responsáveis pelas decisões relacionadas ao tratamento, bem como pela direção adotada quanto às condutas que a eles competem.

Ainda em relação à adesão dos pais ao tratamento, Clarke et al. (2015) analisaram, em uma pesquisa quantitativa, a relação entre a frequência no tratamento e o envolvimento da criança em tarefas de casa dadas aos pais como possíveis promotores de melhores resultados no tratamento. A pesquisa evidenciou que o envolvimento ativo com o tratamento do filho é mais importante na previsão da resposta à intervenção do que a frequência 
das sessões. Deste modo, um esclarecimento prévio aos pais sobre aspectos do TDAH e sua inclusão na elaboração do plano de tratamento parece, de acordo com as pesquisas, repercutir de forma bastante favorável no tratamento.

Portanto, os estudos abrangidos nesta categoria permitem identificar que os pais podem ser responsáveis pelas primeiras tratativas feitas em relação às opções de tratamento. As informações de que puderem dispor para esta escolha serão relevantes para o curso do tratamento, mesmo que muitos pais precisem optar sem necessariamente terem informações disponíveis. Neste sentido, as opções puramente medicamentosas ainda são as mais difundidas e aderidas pelos pais. No entanto, os pais necessitam muitas vezes do auxílio dos profissionais para serem orientados quanto à condução do tratamento e qual sua participação nele. A adesão efetiva ocorrerá a partir do entendimento dos pais acerca da importância dos aspectos do tratamento e da implicação destes com o processo.

\section{Estudos que investigaram a eficácia para intervenções com pais como alternativa de tratamento do TDAH nos filhos}

Os resultados encontrados nesta categoria apresentam estudos que avaliaram a eficácia de intervenções com pais como parte do tratamento da criança com TDAH, demonstrando que há uma ampla gama de opções disponíveis (e pouco conhecidas) para que os pais possam participar do tratamento dos filhos. Os estudos aqui descritos incluem, predominantemente, abordagens comportamentais especialmente as de caráter de treinamento de habilidades, psicoeducação comportamental ou até mesmo iniciativas que incluem práticas como a da atenção plena, oferecidas aos pais nas modalidades grupal ou individual e que tem demonstrado resultados positivos no tratamento de crianças com TDAH (Coates et al., 2015; Daley \& O’Brien, 2013; Mulqueen et al., 2015; Stattin et al., 2015).

Um estudo empírico conduzido na Suécia (Stattin et al., 2015) avaliou a eficácia de quatro programas de treinamento de pais para crianças com problemas de externalização. Dentre os programas em estudo, três eram comportamentais, sendo um deles um programa não-comportamental 
voltado para a redução de problemas de comportamento infantil e sintomas de transtorno de déficit de atenção/hiperatividade (TDAH), para a melhoria da parentalidade e competência parentais, para a diminuição de comportamentos parentais negativos, estresse parental e sintomas depressivos.

Os benefícios da inclusão dos pais como possibilidade de redução dos sintomas de TDAH nas crianças foram avaliados por meio de uma revisão sistemática de ensaios clínicos realizados por Coates, Taylor, e Sayal (2015). Os autores analisaram onze ensaios randomizados e não randomizados, mas que possuíam o grupo de controle para crianças com TDAH. As intervenções parentais foram associadas à redução sintomas de TDAH e mostraram-se também eficazes para problemas de conduta comórbidos, muito freqüentemente diagnosticados com o TDAH.

Resultados semelhantes e igualmente positivos para intervenção com pais foram encontrados em uma meta-análise realizada por Mulqueen, Bartley, e Bloch (2015) através da análise de oito ensaios experimentais sobre a eficácia das intervenções para pais no tratamento do TDAH em crianças pré-escolares como alternativa ao tratamento medicamentoso. Seis dentre os oito ensaios compararam um programa de treinamento de pais a uma lista de espera de tratamento habitual; um dos ensaios comparou um programa de treinamento de pai e filho combinado com a condição de lista de espera e, por fim, o outro ensaio comparou um programa combinado de treinamento para pais e consulta escolar com uma condição de controle de tratamento comunitário.

Os oito ensaios totalizaram uma média de 399 participantes. Constatou-se que houve um benefício significativo para a criança através das intervenções comportamentais parentais comparando com condições de controle. Deste modo, a meta-análise forneceu evidências de que as intervenções parentais são eficazes para o tratamento de TDAH em pré-escolares (Mulqueen et al., 2015)

Outro estudo que forneceu dados positivos quanto a intervenções com pais foi relatado por Neece (2014). Através do estudo empírico foi examinada a eficácia da redução do estresse focado na atenção plena para pais de crianças com problemas de desenvolvimento que incluíam o TDAH. Participaram do estudo 46 pais de crianças com problemas de desenvolvimento, 
sendo aleatoriamente designados para tratamento imediato ou grupo de lista de espera. Como resultado, os pais relataram redução significativa do estresse e dos sintomas de depressão, além de maior satisfação com a vida em comparação com lista de espera/controle, também houve menos problemas de comportamento após a intervenção, especificamente nas áreas de problemas de atenção e sintomatologia do TDAH.

Além das modalidades observadas de interação pai-filhos, os treinamentos de habilidades e atenção plena, e os programas de autoajuda para pais podem significar a possibilidade de aumentar recursos parentais para lidar com o TDAH nas crianças. Esta constatação foi o resultado de um estudo desenvolvido por Daley e O'Brien (2013), no qual foi avaliada a eficácia de um programa de treinamento de autoajuda para pais de crianças com TDAH. A intervenção possibilitou a redução dos sintomas de TDAH em $45 \%$ das crianças envolvidas no processo. Ainda, constatou-se que a competência dos pais aumentou, refletindo que os conhecimentos desenvolvidos nas intervenções passaram a desempenhar um papel importante na qualidade de vida da família (Daley \& O’Brien, 2013).

Em síntese, os estudos relatados neste tópico apresentam evidências significativas do benefício de intervenções com foco no melhor desempenho da parentalidade ou na redução de estresse como parte de um conjunto de intervenções visando benefícios para as crianças com TDAH. Entretanto, apesar das evidências gradualmente consistentes quanto à importância de incluir os pais como parte do tratamento de crianças com TDAH, ainda existem muitas barreiras que impedem o acesso dos pais a estas intervenções.

\section{As barreiras e os acessos aos pais em intervenções que os incluem como parte do tratamento da criança com TDAH}

Estudos apontam que apesar da diversidade de intervenções que incluem pais para o tratamento de TDAH, algumas barreiras podem dificultar que os pais se beneficiem destas, especialmente quanto à supervalorização de medidas exclusivamente medicamentosas em detrimento de medidas psicossociais, divergências não dialogadas de pais e profissionais 
quanto a percepções e conduções do quadro, assim como o efeito negativo de estresse parental ou presença de psicopatologia parental. Por outro lado, há fatores que são apontados como facilitadores, dentre eles a tomada de conhecimento dos pais quanto a benefícios de tratamentos que não privilegiem apenas medicação (que são frequentemente facilitadas pelos profissionais que realizam o atendimento inicial da criança), bem como medidas de acompanhamento de saúde e possibilidades mentais dos pais para desempenhar seu papel nas intervenções terapêuticas. Sendo assim, barreiras e acessos demandam uma avaliação quanto à interferência que podem exercer no tratamento de crianças diagnosticas com TDAH.

Um estudo qualitativo realizado no Reino Unido avaliou a percepção de 25 pais e 18 profissionais sobre programas parentais atualmente disponíveis para crianças pré-escolares diagnosticadas com TDAH e constatou que existe atualmente um reconhecimento acerca da importância de intervenções precoces que incluam os pais no processo terapêutico. Estas intervenções têm sido cada vez mais consideradas e utilizadas, mesmo que muitas vezes tais programas não sejam avaliados adequadamente quanto a demandas e conteúdos específicos relacionados ao TDAH (Smith et al., 2015). Neste estudo foram realizadas entrevistas semi-estruturadas com os pais a fim de identificar barreiras e facilidades associadas ao acesso e eficácia dos programas voltados para pais. Como resultado, observou-se que muitos temas trabalhados nos programas eram mais genéricos para problemas de comportamento disruptivo predominantemente.

Ainda tratando do estudo citado, os objetivos comuns dos programas eram focalizados no incremento da motivação dos pais, provocando mudanças nas práticas destes através de intervenções voltadas às necessidades parentais mais frequentes (por exemplo, em relação à autoconfiança, depressão ou TDAH parental). Uma barreira verificada foi a diferença de pontos de vista de pais e profissionais, especialmente em relação a expectativas de tratamento, sugerindo que esta deve ser uma via a explorar, afim de proporcionar aos envolvidos no processo uma melhor efetividade dos programas que enfocam intervenções com os pais (Smith et al., 2015).

Outra barreira, segundo Neece (2014), são os elevados níveis de estresse de pais de crianças com transtornos no desenvolvimento, em 
contraste com pais de crianças com desenvolvimento típico. O estresse apresentado pelos pais pode impactar negativamente no convívio com a criança e em seu comportamento. Mesmo com esta constatação, são escassas as intervenções com o objetivo de diminuir o problema da situação emocional dos pais. A possibilidade de dificuldades mentais, cognitivas ou psicopatologias nos pais é também uma importante barreira em relação ao tratamento de crianças com TDAH, seja através da negação da psicopatologia apresentada nos filhos ou na dificuldade em participar ativamente dos manejos que aos pais competem no tratamento (Neece, 2014).

Evidências sugerem que o TDAH parental, especialmente quando não diagnosticado, pode se apresentar como uma barreira ao tratamento da criança com TDAH, ao mesmo tempo que reduz a eficácia de um tratamento, seja ele farmacológico ou de treinamento comportamental dos próprios pais (Wang et al., 2014). Embora a literatura sobre estas questões ainda se apresente reduzida e muitas vezes pouco consistente, estudos sugerem que o tratamento dos sintomas de TDAH, quando o quadro existe nos pais, pode ser suficiente para ocasionar melhorias desejadas nos resultados parentais e comportamentais da criança. Portanto, as intervenções dirigidas ao TDAH apresentado por pais e filhos, quando ambos estão presentes, são provavelmente necessárias para melhorar as interações entre eles, bem como o funcionamento familiar.

As percepções parentais podem também potencializar ou inviabilizar o tratamento. Desse modo, avaliar os pontos que são valorizados pelos pais em relação a um tratamento ajuda a estabelecer que aspectos foram considerados por estes e de que forma isso repercute no seguimento da opção terapêutica desejada. Zima, Bussing, Tang, e Zhang (2013) através de estudo longitudinal, controlado, realizado com 529 crianças que recebiam algum tipo de cuidado no intervalo de seis meses de tratamento para TDAH, relataram que quando se avaliam as percepções parentais de barreiras, o conhecimento do TDAH e a disposição de aconselhamento prévio para a permanência da criança sob cuidados, somente a disposição para a medicação foi preditiva para a continuidade no tratamento. Essa supervalorização do tratamento medicamentoso pode ser considerada preocupante, pois desconsidera outras inciativas que possam ser eficazes. Além disso, 
Mulqueen et al. (2015) ressaltam que, embora os psicoestimulantes sejam muito frequentemente utilizados para tratar TDAH, é recomendável cautela em relação aos efeitos colaterais em crianças mais jovens.

Edwards e Howlett (2013), com base na análise de doze entrevistas semi-estruturadas com pais e profissionais ativos na área de tratamento do TDAH, demonstram como as intervenções dos pais têm procurado expandir o domínio terapêutico do TDAH para além do domínio exclusivo de biopsiquiatria. Assim, a inconsistência nos estudos que se propõem a investigar a efetividade de ações com pais como parte do tratamento da criança pode também ser considerada uma barreira em um contexto no qual as evidências ainda são primordiais.

Neste sentido, uma pesquisa conduzida por Zwi, Jones, Thorgaard, York, e Dennis (2011) analisou cinco estudos com 284 participantes que comparavam o treinamento dos pais ao tratamento de costume para TDAH. Quatro desses estudos focalizaram nos problemas de comportamento das crianças e um deles avaliou mudanças nas habilidades de parentalidade. Dos quatro estudos que visam o comportamento das crianças, dois se concentraram no comportamento do infante em casa e dois focaram no comportamento da criança na escola. Os dois estudos focalizando o comportamento em casa apresentaram conclusões diferentes: um deles não encontrou nenhuma diferença entre o treinamento dos pais e o tratamento como de costume, enquanto o outro relatou resultados estatisticamente significativos para o treinamento dos pais versus o grupo controle.

Ainda sobre o estudo de Wzi et al. (2011), os dois estudos que trataram do comportamento escolar da criança também tiveram achados diferentes: um deles não encontrou diferença entre os grupos, enquanto o outro relatou resultados positivos para o treinamento dos pais quando o TDAH não era comórbido com outro transtorno externalizante, além de melhora para as meninas e crianças com medicação. Deste modo, apesar da falta de consistência de alguns estudos em função de má qualidade metodológica e do risco de viés nos resultados, o treinamento parental tem se mostrado eficaz na redução do estresse e aumento de habilidades parentais.

Superar a dificuldade das barreiras e dos acessos aos diferentes tipos de terapêuticas parece ser um desafio ainda presente no trajeto a 
ser cumprido para legitimar os tratamentos que visam incluir os pais no processo, pois, mesmo que as evidências disponíveis sejam limitadas e algumas descobertas possam ser passíveis de interpretação, o papel positivo da psicoeducação com pais e outras intervenções em crianças e adolescentes com TDAH é suportada pela maioria da literatura disponível sobre o tema (Montoya, Colom, \& Ferrin, 2011). Entretanto, mesmo com tais evidências, há ainda pouca adesão de pais a estas intervenções (Stahlschmidt, Threlfall, Seay, Lewis, \& Kohl, 2013).

\section{DISCUSSÃO}

Os artigos pesquisados sugerem efeitos positivos quando as intervenções oferecidas aos pais são ofertadas como parte do tratamento das crianças. Tais achados apoiam as diretrizes da prática clínica e sugerem que intervenções parentais podem ser eficazes (Coates et al., 2015; Daley \& O’Brien, 2013; Mulqueen et al., 2015; Stattin et al., 2015). Os estudos que compõe este trabalho tiveram o propósito de avaliar a eficácia de intervenções parentais e apresentaram resultados positivos quanto a essas intervenções, sejam elas das modalidades psicossociais, de atenção plena ou combinada. Tal constatação é suficiente para sugerir como necessária a garantia da disponibilidade de intervenções parentais na comunidade frente aos benefícios constatados. Entretanto, Coates et al. (2015) afirmam que, embora evidências sugiram que os programas parentais podem melhorar a qualidade dos relacionamentos pai-filho, ainda é escassa a procura e participação nestes programas.

Barreiras importantes devem ser analisadas e minimizadas para que os pais possam ter alcance a estas modalidades de tratamento. As barreiras de acesso dos pais às modalidades de tratamento que visam à intervenção parental, relatadas nos estudos, parecem estar relacionadas a fatores como falta de informação dos pais quanto aos benefícios proporcionados por estas intervenções ou, até mesmo, algo mais basal como a falta de motivação para conhecer aspectos do transtorno apresentado pelos filhos e os melhores recursos de tratamento disponíveis para tratamentos. A existência de limitações cognitivas ou impostas pela existência de quadros 
psicopatológicos nos pais, se estes não estiverem recebendo tratamento adequado, pode representar limitações quanto a um bom desempenho no tratamento das crianças.

A baixa consistência dos estudos e o pouco acesso dos pais à divulgação dos resultados podem limitar a procura e a adesão deles a modalidades de tratamento que incluam sua participação, visto que acabam por não desenvolverem a consciência da importância desta implicação ou por sustentarem a crença de que o tratamento exclusivamente medicamentoso é mais eficaz. Mesmo as intervenções direcionadas aos pais estando gradativamente mais difundidas, programas de treinamento podem ter deficiências quanto aos conteúdos abordados, que muitas vezes são genéricos ou mais relacionados a comportamentos disruptivos não tão específicos como os do TDAH, como aqueles voltados para questões de autoconfiança, depressão ou TDAH parental.

Outra dificuldade apontada pelos estudos parece ser a supervalorização dos pais em relação ao tratamento farmacológico. Este é um problema que reflete padrões atuais de diagnóstico e tratamento frente à crença de que a medicação pode, por si só, representar a cura de uma doença ou transtorno ou, ainda, ser mais efetiva que o tratamento psicossocial ou combinado. Assim, cabe aos profissionais intervir e evitar as desistências, auxiliar nas dificuldades dos cuidadores e desconstruir estereótipos de comportamentos e de tratamentos disseminados pelas instituições e pela sociedade em geral. Além disso, cabe ainda ao profissional atentar para os principais agentes de mudanças: a família e a própria criança, despertando na família a capacidade de buscar informações e discutir a forma como o problema do filho é tratado.

Em relação a acessos, a abordagem dos pais pelos profissionais responsáveis pelos primeiros manejos relacionados ao TDAH, sejam eles psiquiatras, médicos, psicólogos ou até mesmo professores, parece oferecer possibilidades que estes pais poderão conhecer e delas se beneficiar. Até mesmo, para que os pais possam se tornar ativos no processo de criação de conhecimento e saber que venha contribuir para o tratamento do transtorno. 


\section{CONSIDERAÇÕES FINAIS}

Os artigos avaliados nesse estudo apresentam informações importantes sobre como estão sendo elaboradas e executadas alternativas de tratamento frente aos prejuízos advindos do TDAH para crianças e suas famílias. Os resultados, ainda que não representem um grande número de publicações sobre o tema, são consistentes quanto aos diversos benefícios da participação dos pais como parte do tratamento da criança com TDAH. As formas de inserção dos pais no tratamento vão além da psicoterapia e se constituem como diferentes perspectivas em relação às intervenções planejadas. Nos tratamentos estudados são oferecidos grupos terapêuticos para pais em separado da criança; grupos de formação e orientação para os pais, abordando as principais características do transtorno e quais os manejos possíveis relacionados ao TDAH; são, ainda, apresentadas outras abordagens para sessões com os pais junto da psicoterapia voltada para a criança.

Assim as intervenções de cunho psicossocial podem auxiliar na qualidade de vida das crianças com TDAH, especialmente se houver uma integração dos elementos que fazem parte do dia a dia da criança, como casa e escola e/ou pais e professores. Muitos tratamentos voltados ao TDAH ainda parecem oferecer vias que visam benefícios únicos, como os de viés medicamentoso, apesar das diretrizes para saúde em muitos países já incluírem como linha de tratamento para o TDAH as intervenções com os pais. A eficácia das abordagens combinadas (pais, crianças, escola e medicação) também deve ser melhor avaliada para que, se comprovados os seus benefícios, sejam mais bem divulgadas, a fim de maximizar ganhos no tratamento para crianças com TDAH. Barreiras configuradas pela falta de acesso dos pais ao conhecimento sobre as diversas formas de tratamento e seus benefícios para a criança com TDAH devem ser pesquisadas por profissionais da área e desfeitas, uma vez que podem ser consideradas entraves para que os responsáveis conheçam, aceitem e utilizem-se dessas intervenções como forma de tratamento eficaz para os infantes.

Aspectos como a abordagem dos pais por um profissional logo no diagnóstico do quadro parecem favorecer a adesão e a implicação destes 
ao longo do tratamento, uma vez que os instrumentaliza para realizar a escolha das melhores conduções para o quadro apresentado pelo filho. Neste sentido, a observação das condições de saúde mental dos pais também pode ser considerada relevante no tratamento da criança que manifesta TDAH, posto que, como grandes referências que são para os infantes, é intensa a influência do funcionamento subjetivo parental no desenvolvimento das crianças. Tem sido comum pais que apresentam dificuldades de administrar o tratamento do TDAH de seus filhos por também apresentarem o quadro clínico, de modo que tais psicopatologias merecem atenção e intervenção, frente a possibilidade de eclosão de diversos aspectos psicopatológicos no desenvolvimento infantil.

Deste modo, pensar o tratamento da criança com TDAH requer observar a dinâmica particular da família, suas dificuldades e potencialidades, como o primeiro e mais profícuo espaço de convívio da criança, de modo que possa se estabelecer um tratamento que ofereça ações em consonância com as particularidades do quadro, bem como da família, dentre as variadas possibilidades de atendimento a esta demanda. A inclusão dos pais no tratamento da criança parece ser um ponto chave no processo, de modo que proporcione para a criança receber respaldo tanto pela via de seu momento terapêutico, como pela amplificação das potencialidades dos pais, seja pelo treinamento destes em relação às características e possíveis manejos do transtorno, ou por um espaço que ofereça reflexão sobre o que lhes será exigido e sobre o que tem como questões a propor sobre seu papel na relação com seu filho. O envolvimento da família no tratamento da criança com TDAH permite a potencialização dos resultados, tendo em vista características particulares de cada quadro na elaboração e execução do plano de tratamento.

\section{REFERÊNCIAS}

American Psychiatric Association (2014). Manual diagnóstico e estatístico de transtornos mentais - DSM-5 (5a ed.; M. I. C. Nascimento, Trad.). Porto Alegre, RS: Artmed. 
Banaschewski, T., Becker, K., Döpfner, M., Holtmann, M., Rösler, M., \& Romanos, M. (2017). Attention-deficit/hyperactivity disorder: a current overview. Deutsch Arzteblatt International, 114 (9), 149-159. doi: 10.3238/arztebl.2017.0149

Barkley, R. A. (2002). Transtorno de déficit de atenção/hiperatividade (TDAH). Porto Alegre, RS: Artmed.

Brinkman, W. B., Hartl M. J., Poling, L. M., Shi, G., Zender, M., Sucharew, H., ... Epstein, J. N. (2013). Shared decision-making to improve attention-deficit hyperactivity disorder care. Patient Education and Counseling, 93(1), 95-101. doi: 10.1016/j.pec.2013.04.009

Brown, T. E. (2007). Transtorno de déficit de atenção: a mente desfocada em crianças e adultos. Porto Alegre, RS: Artmed.

Caliman, L. V. (2010). Notas sobre a história oficial do transtorno do déficit de atenção/hiperatividade TDAH. Psicologia: Ciência e Profissão, 3o(1), 45-61. doi: 10.1590/S1414-98932010000100005

Clarke, A. T, Marshall, S. A, Mautone, J. A, Soffer, S. L, Jones, H.A., Costigan, T. E., ... Power, T. J. J. (2015). Parent attendance and homework adherence predict response to a family-school intervention for children with ADHD. Journal of Clinical Child \& Adolescent Psychology, 44(1), 58-67. doi: 10.1080/15374416.2013.794697

Coates, J., Taylor, J. A., \& Sayal, K. J. (2015). Parenting interventions for ADHD: a systematic literature review and meta-analysis. Journal of Attention Disorders, 19(10), 831-843. doi: 10.1177/1087054714535952

Daley, D., \& O'Brien, M. (2013). A small-scale randomized controlled trial of the self-help version of the New Forest Parent Training Programme for children with ADHD symptoms. European Child \& Adolescent Psychiatry, 22(9), 543-552. doi: 10.1007/s00787-013-0396-8

Dallos, R., \& Smart, C. (2011). An exploration of family dynamics and attachment strategies in a family with $\mathrm{ADHD} /$ conduct problems. Clinical Child Psychology and Psychiatry, 16(4), 535-550. doi: 10.1177/1359104510387391

Edwards, C., \& Howlett, E. (2013). Putting knowledge totrial: 'ADHD parents' and the evaluation of alternative therapeutic regimes. Social Science \& Medicine, 81, 34-41. doi: 10.1016/j.socscimed.2013.01.015 
Folquitto, C. T. F. (2013). Desenvolvimento psicológico e estratégias de intervenção com crianças com diagnóstico de TDAH (Tese de doutorado). Instituto de Psicologia, Universidade de São Paulo, São Paulo.

França, M. T. D. B. (2012). Transtorno de déficit de atenção e hiperatividade (TDAH): ampliando o entendimento. Jornal de Psicanálise, 45(82), 191-207. Recuperado de: http://pepsic.bvsalud.org/scielo. php?script=sci_arttext\&pid=So103-58352012000100014

García, M. G., Tato, L. P., Borbujo, J. S., Corral, L. M., Fabián, A. H., \& Martín, L. S. F. (2008). Trastorno por déficit de atención e hiperactividad: un problema actual. Anales de Pediatría, 69(3), 244-250. doi: 10.1157/13125819

Gusmão, M. M. G. (2009). Comportamento infantil conhecido como hiperatividade: consequência do mundo contemporâneo ou $T D A H$ ? (Dissertação de mestrado). Universidade Federal de Minas Gerais, Belo Horizonte.

Johnston, C., \& Mash, E. (2001). Families of children with attention deficit/ hyperactivity disorder: review and recommendations for future research. Clinical Child and Family Psychology Review, 4(3), 183-207. doi: 10.1023/A:1017592030434

Keown, L. J. (2012). Predictors of Boys' ADHD symptoms from early to middle childhood: the role of father-child and mother-child interactions. Journal of Abnormal Child Psychology, 40(4), 569-581. doi: 10.1007/s10802-011-9586-3

Leite, W. (2002). TDAH: Estudo de prevalência em estudantes da primeira a quarta série do Ensino Fundamental do Município de Inhaúma-MG (Monografia de Especialização). Universidade Federal de Minas Gerais, Belo Horizonte.

McGuinness, T. M. J. (2008). Helping parents decide on ADHD treatment for their children. Journal of Psychosocial Nursing and Mental Health Services, 46(8), 23-27. Recuperado de: https://www.ncbi. nlm.nih.gov/pubmed/18777965 
Modesto-Lowe, V., Chaplin, M., Godsay, V., \& Soovajian, V. (2014). Parenting teens with attention-deficit/hyperactivity disorder: challenges and opportunities. Clinical pediatrics, 53(10), 943-948. doi: 10.1177/ooog922814540984

Montoya, A., Colom, F, \& Ferrin, M. (2011). Is psycho education for parents and teachers of children and adolescents with ADHD efficacious? A systematic literature review. European Psychiatry, 26(3), 166-175. doi: 10.1016/j.eurpsy.2010.10.005

Moreno Fontiveros, M., Martínez Vera, M., Tejada González, A., González Igeño, V., \& García Resa, O. (2015). Actualización en el tratamiento del trastorno del déficit de atención con/sin hiperactividad (TDAH) en Atención Primaria. Revista Clínica de Medicina de Familia, 8(3), 231-239. doi: 10.4321/S1699-695X2015000300006

Mulqueen, J. M., Bartley, C. A., \& Bloch, M. H. J. (2015). Meta-analysis: parental interventions for preschool ADHD. Journal of Attention Disorders, 19(2), 118-124. doi: 10.1177/1087054713504135

Murray-Close, D., Hoza, B., Hinshaw, S. P., Arnold, L. E., Swanson, J., Jensen, P. S., ... Wells, K. (2010). Developmental processes in peer problems of children with attention-deficit/hyperactivity disorder in The Multimodal Treatment Study of Children With ADHD: developmental cascades and vicious cycles. Development and Psychopathology, 22(4), 785-802. doi: 10.1017/So954579410000465

Neece, C. L. (2014). Mindfulness-based stress reduction for parents of young children with developmental delays: implications for parental mental health and child behavior problems. Journal of Applied Research in Intellectual Disabilities, 27(2), 174-186. doi: 10.1111/jar.12064

Nussbaum, A. M. (2015). Guia para o exame diagnóstico segundo o DSM-5. Porto Alegre, RS: Artmed.

Pfiffner, L. J., Hinshaw, S. P., Owens, E., Zalecki, C., Kaiser, N. M., Villodas, M., \& McBurnett, K. (2014). A two-site randomized clinical trial of integrated psychosocial treatment for ADHD-inattentive type. Journal of Consulting and Clinical Psychology, 82(6), 11151127. doi: 10.1037/aoo36887 
Rohde, L. A., \& Halpern, R. (2004). Transtorno de déficit de atenção/ hiperatividade: atualização. Jornal de Pediatria, 8o(2), 61-70. doi: 10.1590/Soo21-75572004000300009

Roskam, I., Stievenart, M., Tessier, R., Muntean, A., Escobar ,M. J., Santelices, M. P., ... Pierrehumbert, B. (2013). Another way of thinking about ADHD: the predictive role of early attachment deprivation in adolescents' level of symptoms. Social Psychiatry Psychiatric Epidemiology, 49(1), 133-144. doi: 10.1007/ s00127-013-0685-Z

Schatz, N. K, Fabiano, G. A, Cunningham, C. E., dosReis, S., Waschbusch, D. A, Jerome, S., ... Morris, K. L. P. (2015). Systematic review of patients' and parents' preferences for ADHD treatment options and processes of care. The Patient, 8(6), 483-497. doi: 10.1007/s40271-015-0112-5 Smith, E., Koerting, J., Latter, S., Knowles, M. M., McCann, D. C., Thompson, M., \& Sonuga-Barke, E. J. (2015). Overcoming barriers to effective early parenting interventions for attention-deficit hyperactivity disorder (ADHD): parent and practitioner views. Child: Care, Health and Development, 41(1), 93-102. doi: 10.1111/cch.12146 Stahlschmidt, M. J., Threlfall, J., Seay, K. D., Lewis, E. M., \& Kohl, P. L. (2013). Recruiting fathers to parenting programs: advice from dads and fatherhood program providers. Children and Youth Services Review, 35(10), 1734-1741. doi: 10.1016/j.childyouth.2013.07.004

Stattin, H., Enebrink, P., Özdemir, M., \& Giannotta, F. ( 2015). A national evaluation of parenting programs in Sweden: The short-term effects using an RCT effectiveness design. Journal of Consulting and Clinical Psychology, 83(6), 1069-1084. doi: 10.1037/aoo39328

Webster-Stratton, C., Reid, M. J., \& Beauchaine, T. P. J. (2013). One-year follow-up of combined parent and child intervention for young children with ADHD. Journal of Clinical Child \& Adolescent Psychology, 42(2), 251-261. doi: 10.1080/15374416.2012.723263

Zima, B. T., Bussing, R., Tang, L., \& Zhang, L. (2013). Do parent perceptions predict continuity of publicly funded care for attention-deficit/ hyperactivity disorder? Pediatrics, 131(1), S50-S59. doi: 10.1542/ peds.2012-1427f 
Zwi, M., Jones, H., Thorgaard, C., York, A., \& Dennis, J. A. (2011). Parent training interventions for Attention Deficit Hyperactivity Disorder (ADHD) in children aged 5 to 18 years. Cochrane Database of Systematic Reviews, (12), 1-73. doi: 10.1002/14651858.CDoo3018. pub3

Wang, C. H., Mazursky-Horowitz, H., \& Chronis-Tuscano, A. (2014). Delivering evidence-based treatments for child attentiondeficit/hyperactivity disorder (ADHD) in the context of parental ADHD. Current psychiatry reports, 16(10), 474. doi: 10.1007/ s11920-014-0474-8 